\title{
Automatic Design of Synchronous Reluctance Motors focusing on Barrier Shape Optimization.
}

\author{
G. Pellegrino, Senior Member, IEEE, F. Cupertino, Senior Member, IEEE, and C. Gerada, Member, IEEE
}

\begin{abstract}
The automated design of Synchronous Reluctance motors based on Multi-Objective, Genetic Optimization and Finite Element Analysis is considered in this paper. Three types of barrier shapes are considered, all described by an effective, limited set of input variables. The three solutions are investigated to establish which of the geometries can give the best torque output and also which one represents the best compromise between output performance and computational time. The analysis presented in this paper shows that Synchronous Reluctance motors designed automatically can give a good performance, can be designed in a reasonable time and it is also shown that not all design degrees of freedom are useful in terms of motor performance. Two prototypes of automatically designed machines have been fabricated and experimentally compared to a third prototype designed according to state-of-the-art design principles.
\end{abstract}

Index Terms - AC Machines, AC Motors, AC Drives, Synchronous Motor Drives, Synchronous Reluctance Machines, Rotor Design, Design optimization, Pareto Optimization, Finite Element Analysis.

\section{INTRODUCTION}

$S^{y}$ ynchronous Reluctance (SyR) motors are a viable alternative to inverter-driven Induction Motors (IM) due to their higher efficiency, lower rotor temperature and their higher transient overload capability. SyR motors have been studied comprehensively in the 1990s [1-5] and recently reconsidered by major manufacturers [6]. In addition, they are the basis for permanent magnet- (PM-) assisted SyR motors, which are a class of Interior PM (IPM) machines of particular interest for their reduced PM quantity [7-8].

The design of transverse laminated SyR rotors with multiple flux barriers has been formalized through the years by many authors. Yet, a standard design approach is an open challenge, in particular for industrial applications. Finite element analysis (FEA) is adopted by all authors, including the ones that base the design on analytical models [9-11]. This is mainly due to the impact of magnetic saturation which is significant and consequently linear magnetic models are inaccurate.

Finite element based design of SyR motors through artificial intelligence techniques is discouraged due to the long simulation times as a result of the numerous FEA evaluations demanded by the search algorithms. This is the case for any kind of optimization algorithim (OA) applied to this motor type as a result of the combination of the high number of candidate solutions and the non-negligible time for FEA evaluation for each candidate. On one hand, the OA will require a number of tentative motor design evaluations depending on the algorithm and on the proper conditioning of the problem (choice of the input variables and selection of the optimization goals). On the other hand, SyR machines tend to require many FEA runs for their performance to be evaluated. For example, Surface mounted PM machines can be evaluated quite comprehensively via a single static FEA simulation $[12,13]$, which is not the case here.

Returning to the number of evaluations required by the $\mathrm{OA}$ to converge, the set of geometric parameters describing the multi-barrier rotor plays a key role. In the literature, this varies and it is generally high and proportional to the number of layers [14].

In previous work, the choice and compromise between the variables for a good description of the rotor geometry and for a fast FEA evaluation of the candidate motors were addressed [15]. A two-step procedure for a time efficient multi-objective genetic algorithm (MOGA) was proposed in [16], having as output a front of SyR motor designs which are Pareto-optimized in terms of torque and torque ripple. In [17] other types of barriers were considered, as an alternative to the circular barriers of $[15,16]$.

This paper further investigates the compromises to be had in choosing a barrier geometry which is more suitable for automatic design. Three-layer rotors will be considered in this paper and three topological geometries will be defined and used for the automatic design. The first having flux barriers of circular shape and the other two with angled barriers made of straight consecutive segments as illustrated in Fig. 1 and 2. The torque-vs-torque ripple performance obtained with the three geometries is compared along with the respective computational times. The number of geometric variables and their effect on computational time are also investigated. The two-step use of the MOGA introduced in [16] is reconsidered and a new procedure for the final Local-Search refinement is proposed. Finally, the motors designed automatically by the MOGA are compared experimentally with a state-of-the-art motor.

\section{ROTOR GEOMETRIES CONSIDERED}

The 3-layer rotor represented in Fig. 1a has circular or C- 
shaped barriers and it is hence referred to as 3C-type. The rotor in Fig. 1b has the barriers made of straight segments, similar to the shape of a $U$. This one is then named 3U-type. The geometric parameters for the definition of the two types of rotors are defined in Fig. 1 and are basically two per layer: the thickness of the $j$-th barrier is $h c_{\mathrm{j}}$ and the angular position of its end at the airgap is $\Delta \alpha_{\mathrm{j}}$. The $3 \mathrm{U}$ rotors have one more variable, $\Delta x$, accounting for the depth of the barriers radial-wise in perunit. With $\Delta x=0$ the outer barrier degenerates into a I-shaped barrier (Fig. 3a). On the other hand, for $\Delta x=1$, the barriers follow the traces set by three circular barriers defined by the same set of parameters $\left(\Delta \alpha_{123}, h c_{123}\right)$, as represented in Fig. $3 \mathrm{~b}$. The rotor with one I- and two U-barriers in Fig. 3a is indicated from now on with the acronym I2U.

\section{A. Geometric variables and rules for automatic drawing}

As mentioned beforehand, the number of geometric parameters has to be as low as possible so to simplify the optimization problem and to reduce the number of iterations needed for convergence. Both the $3 \mathrm{C}$ and $\mathrm{I} 2 \mathrm{U}$ rotor types account for six variables, while the 3U-type accounts for seven. With regards to the criteria used to draw the rotors according to the six or seven input variables, the preliminary assumptions valid for all the geometries considered are:

1) The ends of the flux barriers are circular, with the diameter equal to the thickness of the respective barrier.

2) The barriers and the flux guides between the barriers have constant thickness throughout their development.

3) All the structural ribs at airgap have the same thickness, preliminarily determined according to fabrication tolerances and centrifugal stress and then verified and refined as necessary with structural FEA at a final design stage.

For all the geometries, the barriers' ends are identified by the three angular coordinates $\Delta \alpha_{123}$. The principles for the construction of the $3 \mathrm{C}$ rotors are shown in Fig. 2. The circular sides of the barriers are centered into the common "center point" defined in the figure. The barriers sides are tangent to the circles that locate the barriers ends. The barriers thicknesses $h c_{123}$ are split into equal parts inwards and outwards of the circular traces represented with dashed lines in Fig. 2, which are derived from the angular inputs $\Delta \alpha_{123}$.

The set of parameters is represented mostly in per-unit, as in the example reported in Table I. The base values of the p.u. angles and heights are the total angle and height available for all the layers. The first angular input $\Delta \alpha_{1}$ is not in p.u. and determines the angular space left to the other angular inputs: the other p.u. angles $\Delta \alpha_{j}\left(j=2\right.$ to $\left.n_{\text {lay }}\right)$ define the layer tips distribution over the remaining part of the half pole angular pitch. Once the barriers tips positions $\Delta \alpha_{123}$ are set, the p.u. thicknesses $h c_{123}$ are defined as follows: if they are all 1 p.u. then the air barriers are all of the same thickness and occupy as much radial space as they can. A minimum thickness of $1 \mathrm{~mm}$ is guaranteed for the steel flux guides in the following examples, and this choice defines the situation of maximum insulation, or maximum air thickness along the $q$ - (quadrature-
) axis of the rotor. The $1 \mathrm{~mm}$ clearance condition avoids overlapping barriers and non-feasible rotors from a manufacturing point of view. The $d, q$ axes are defined in Fig. $3 a$.

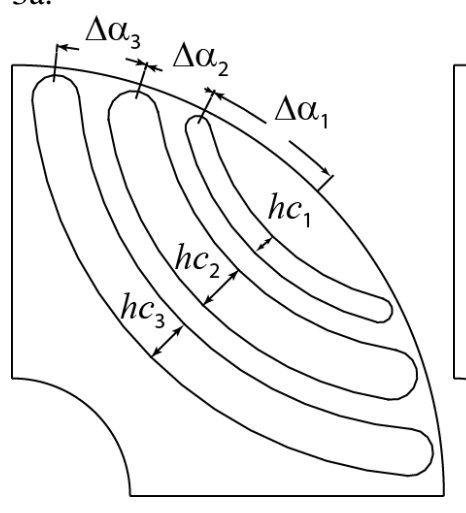

(a)

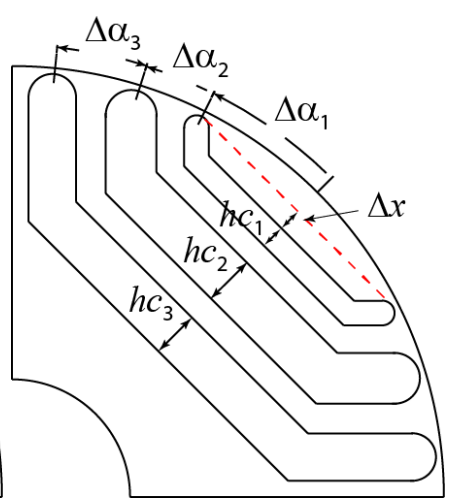

(b)
Fig. 1. Set of parameters defining the $3 \mathrm{C}$ (a) and the $3 \mathrm{U}(\mathrm{b})$ rotor geometries.

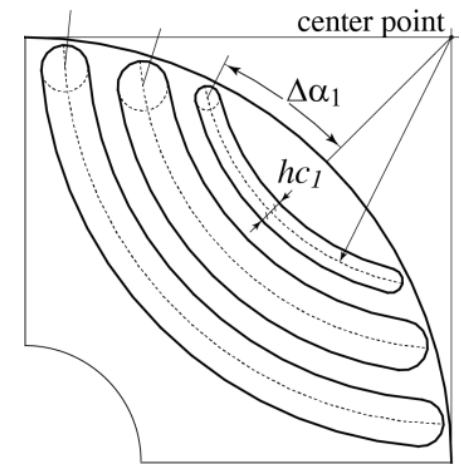

Fig. 2. Principles for the construction of the 3C rotor.

TABLE I

LIMITS OF THE SEARCH SPACE FOR THE Global SEARCH (GS) Optimization STAGE

\begin{tabular}{|c|c|c|c|}
\hline Parameter & $\begin{array}{l}\text { Min } \\
\text { value }\end{array}$ & $\begin{array}{l}\text { Max } \\
\text { value }\end{array}$ & Units \\
\hline$h c_{1}$ & 0.2 & 1 & p.u. \\
\hline$h c_{2}$ & 0.2 & 1 & p.u. \\
\hline$h c_{3}$ & 0.2 & 1 & p.u. \\
\hline$\Delta \alpha_{1}$ & 15 & 27 & degrees \\
\hline$\Delta \alpha_{2}$ & 0.33 & 0.75 & p.u. \\
\hline$\Delta \alpha_{3}$ & 0.33 & 0.75 & p.u. \\
\hline$\Delta x$ & 0 & 1 & p.u. \\
\hline$\gamma$ & 20 & 80 & degrees \\
\hline
\end{tabular}

Returning to the p.u. representation of the input parameters, if all the p.u. heights are at a minimum (e.g. 0.2), then the barriers are again all of the same thickness and $20 \%$ of the previous example. All other situations are combinations of the previous ones.

The construction of both the $3 \mathrm{U}$ geometry and its subcase I2U is derived from the $3 \mathrm{C}$ one, as depicted in Fig. 3. The barrier ends are placed in the same positions as they would have been placed in the $3 \mathrm{C}$ rotor. Once more, the barriers have constant thickness. The thickness of the steel flux guides is calculated as if the barriers where circular and then applied to 
the "U" geometry. In Fig. 3b it is easy to see how the profiles of the $3 \mathrm{U}$ are related to the $3 \mathrm{C}$ geometry, in the $\Delta x=1$ case. The other extreme case $\Delta x=0$ (I2U) is drawn starting from the exterior barrier (barrier number 1) and then going inwards, following the air and steel thickness distribution calculated as for the $3 \mathrm{C}$ rotor. The generic $3 \mathrm{U}$ case is in between with $0<\Delta x$ $<1$.

As to the structural ribs and mechanical stress issues, a width of $0.5 \mathrm{~mm}$ was used for all the inter-layer ribs during the optimization. Prior to prototyping all the designed prototypes have been analyzed for centrifugal stress loading via structural FEA at the maximum speed of $8000 \mathrm{rpm}$. In comparative terms it is the I2U geometry the one which better withstands centrifugal forces, due to the lower quantity of mass in the peripheral areas of the laminations.

\section{TORQUE AND TORQUE RIPPLE OPTIMIZATION}

\section{A. Fast-FEA evaluation}

The performance indices to be optimized by the MOGA are the torque and the torque ripple. Other potential objectives to be optimized by the MOGA could have been efficiency, total material cost or weight of the active parts [17-18]. These objectives would require more time consuming transient simulations. These would also require an optimization procedure that includes the stator geometry and the stator to rotor split ratio. This is out of scope for the exercise presented in this paper. However, the conclusions drawn here can be translated to any other optimization problem.

For the sake of computational speed, a single current amplitude and a single phase angle condition are simulated in the process of evaluating the optimization candidates. The current amplitude level used in the following examples is $200 \%$ of the continuous operation current: this condition is intermediate between the continuous operation condition $(100 \%)$ and the maximum overload (300\%). The decision of optimizing the machine torque and ripple at overload conditions comes from the results of preliminary investigations which revealed that the machines with good torque ripple figures in overload conditions are likely to have a limited torque ripple also at the lower current levels, but not vice-versa. Machines optimized at low p.u. currents generally have a high torque ripple at overload. For this reason, the $200 \%$ current condition was used here.

Dealing with the current phase angle $\gamma$ in $d-q$ synchronous coordinates, the correct evaluation of the motor torque capability given the current amplitude would require the knowledge of the maximum torque per Ampere (MTPA) phase condition $\left(\gamma_{M T P A}\right)$, corresponding to the $200 \%$ current level used in the evaluation. Instead of repeating the simulation of one motor at different values of $\gamma$ to find the best one, we added the phase angle among the input variables of the MOGA. Each machine is then evaluated at a single current phase angle, which is randomly selected by the MOGA. After the torque-vs-torque ripple optimization is completed, it is verified that:

- all the machines of the Pareto front are correctly evaluated at their respective $\gamma_{\text {MTPA }}$ condition

- the torque ripple is minimized with particular reference around the MTPA condition.

The MOGA is then capable of optimizing the torque for a given ampere value, and this makes the FEA evaluation very quick with no need of repeated tentative values of $\gamma$. In addition, all the Pareto-optimal machines tend to have a minimum-ripple trajectory in the $\left(i_{d}, i_{q}\right)$ plane which fairly coincides with the MTPA trajectory. To the authors' understanding, this is a byproduct of the MOGA based optimization of $\gamma$.

The torque ripple is calculated as the standard deviation of the FEA torque waveform calculated at $n$ equally spaced rotor positions over one stator slot pitch $\left(\tau_{s t}\right)$. One stator slot pitch was chosen as it is representative of the major torque ripple component for distributed winding machines. This idea was first introduced in [15], where the minimum number of rotor position simulations required to avoid significant aliasing of torque harmonics was also discussed. It was shown that five rotor positions equally spaced over the stator slot pitch and with a random offset applied by the MOGA can minimize the fundamental and third torque ripple harmonics with very quick computation. Three examples of torque ripple evaluation are shown in Fig. 4. The torque waveform in the figure does not refer to any of the final designs presented in the paper, and refers to a non-optimal machine with a high per unit ripple for better evidence of the impact of torque sampling on torque ripple evaluation. The introduction of the random offset (Figs. $4 \mathrm{~b}$ and $4 \mathrm{c}$ ) reduces the simulation time per design case at the cost of a more noisy functional evaluation. The same candidate machine design can be evaluated more optimistically (Fig. 4b) or more realistically (Fig. 4c) according to the value of the random offset. Using this technique, the evaluation of one candidate motor consists only of five time-stepped FEA simulations and takes 2.6s on a Intel Xeon E5-1620 workstation (4 cores, $3.60 \mathrm{GHz}, 16 \mathrm{~GB} \mathrm{ram}$ ). This result also takes advantage of the use of multi-core parallel calculation. Parallel computing is possible thanks to the capability of executing multiple instances of FEMM 4.2 [19] in parallel via the "parfor" Matlab [20] command, purposely made for parallel execution of loop iterations. Four to six candidates can be simulated on a standard multicore personal computer, resulting in a significant increase of simulation speed. Computational times are discussed in the following subsection.

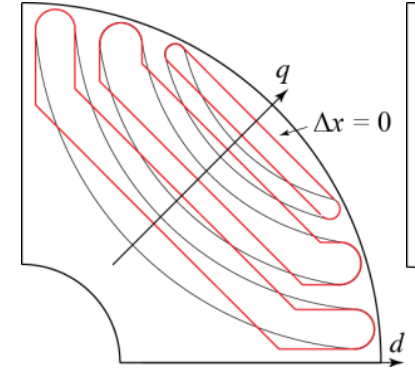

(a)

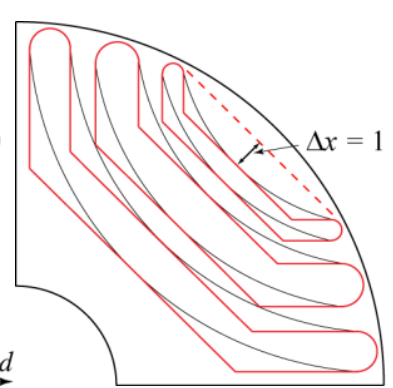

(b)
Fig. 3. Effect of the displacement parameter $\Delta x$ on the $3 \mathrm{U}$ rotors: a) is with $\Delta x=0$ and it is called I2U; b) is with $\Delta x=$ 1 and has the angled barriers in the same radial positions of a $3 \mathrm{C}$ rotor. 

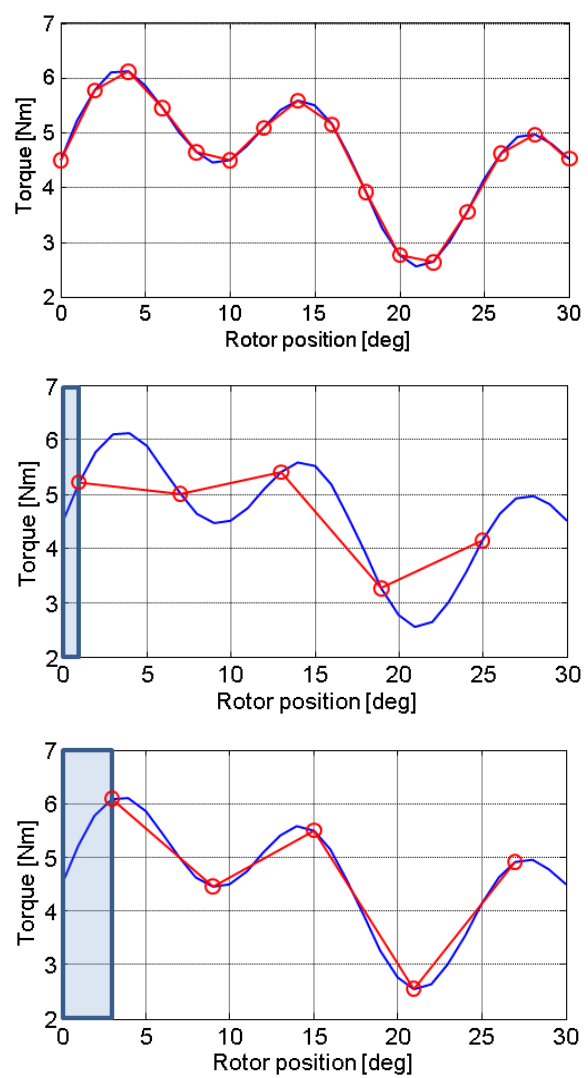

Fig. 4. Evaluation of the torque ripple during optimization: a) with 15 FEA simulations in fixed positions; b) and c) with 5 simulations with random initial position, illustrated by the blue rectangle, selected randomly by the MOGA. b) normal situation (misestimate) and c) "fortunate" situation (ripple estimate nearly correct).

\section{B. Genetic Algorithm optimization procedure}

The MOGA-based design procedure proposed in [16] consists of a first stage called global search (GS) and a successive local search refinement stage (LS). Both stages use the NSGAII algorithm [21] embedded in the Matlab Optimization Toolbox. The result of the GS MOGA is a front of Pareto-non-dominated solutions, from which one motor is selected to be the basis for the successive LS refinement.

The bounds of the GS search space are kept as large as possible, to explore all potential solutions. Table I reports the bound values used for the GS optimization stage.

The quality of the final solution found by the GS-MOGA is related to the amount of time dedicated to the search, which is a function of the number of individuals evaluated by the algorithm to populate its output Pareto front. The number of evaluations is controlled via two main parameters which are the size of the population, accounting for how big is the set of candidate solutions that the $\mathrm{OA}$ evaluates and then manipulates to form an improved population, and the number of generations, that is how many successive times the populations are repeatedly evaluated and manipulated before the final Pareto front is obtained. Roughly speaking, the product of these two numbers indicates the overall number of evaluations processed by the OA. The higher is the number of evaluations, the more likely the optimal Pareto front is the actual set of non- dominated solutions to the problem.

Considering the stochastic nature of genetic algorithms, a single MOGA run cannot guarantee that the actual optimal Pareto front is found as premature convergence to local minimums is always possible. For this reason, it was here chosen to use small-size GS runs which are computationally fast and repeat the GS stage for four times. The GS solutions are then refined via one further LS run. This 4GS-LS approach showed to be much quicker and more consistent than running single GS runs of larger size.

In particular, the four GS runs used in all the examples have a population of 60 individuals and are iterated over 50 generations, corresponding to 3000 functional evaluations. Such a GS run takes nearly 2.5 hours on the processor specified previously. After the four GS runs, the most promising solution is selected from the four Pareto fronts obtained. The selection considers both the machine performance and feasibility. Then the single LS run is executed using search bounds equal to the parameters of the selected GS solution plus or minus $15 \%$ of each input parameter. The overall optimization procedure thus includes 5 optimization runs and takes about 12.5 hours for a total 15,000 evaluations.

In Fig. 5 the best $60 \times 50$ GS Pareto front and the LS Pareto front are represented for the I2U geometry. The selected GS solution and the final LS solution used for the prototype are shown in the figure. The boundaries of the LS input parameters are reported in Table II.

The same 4GS+LS design procedure has been repeated for all rotor geometries: 3C, 3U and I2U.

Tests carried out showed that the results of the 4GS+LS optimization are very similar to the ones obtained with a single GS-MOGA runs of a larger size (200x100), consisting of 20,000 evaluations each. However, one single run is not enough to find the global Pareto front, and two or three 20,000 evaluation runs would have taken 2 to 4 times longer than the proposed $4 \mathrm{GS}+\mathrm{LS}$ procedure.

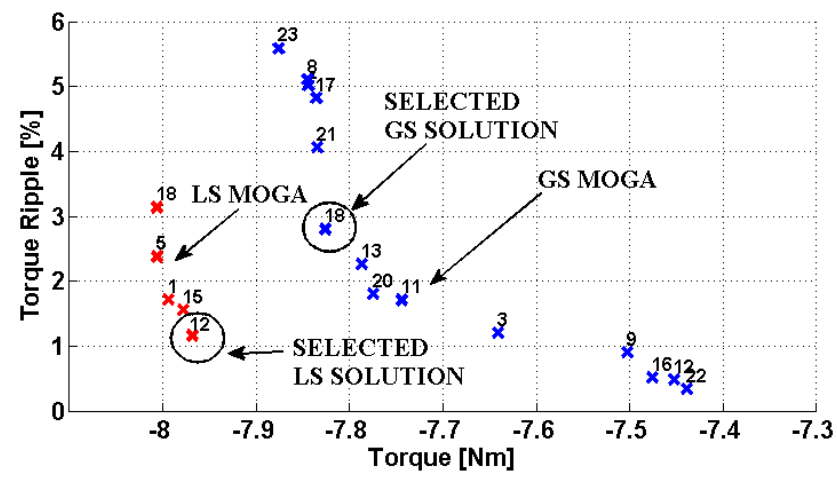

Fig. 5. Detail of one of the GS-MOGA Pareto fronts (blueright) and LS-MOGA (red-left), and solutions selected out of the two fronts. 
TABLE II

LIMITS OF THE SEARCH SPACE FOR THE LOCAL SEARCH (LS) OPTIMIZATION STAGE OF THE I2U ROTOR

\begin{tabular}{llll}
\hline \hline Parameter & $\begin{array}{c}\text { Min } \\
\text { value }\end{array}$ & $\begin{array}{c}\text { Max } \\
\text { value }\end{array}$ & Units \\
\hline$h c_{1}$ & 0.47 & 0.67 & p.u. \\
$h c_{2}$ & 0.40 & 0.72 & p.u. \\
$h c_{3}$ & 0.32 & 0.52 & p.u. \\
$\Delta \alpha_{1}$ & 18 & 20.5 & degrees \\
$\Delta \alpha_{2}$ & 0.50 & 0.67 & p.u. \\
$\Delta \alpha_{3}$ & 0.60 & 0.75 & p.u. \\
$\gamma$ & 60 & 70 & degrees \\
\hline \hline
\end{tabular}

\section{Results of the Automatic Design}

\section{A. Optimized geometries}

The results of the 4GS+LS application of the MOGA are reported in Fig. 6 for the three geometries. The angular positions and thicknesses of the barriers of all the rotors are very similar. The positions of the equivalent rotor "slots" are evidenced by red circles. Moreover, the barriers of $3 \mathrm{U}$ tend to be close to a I2U-like rotor, meaning the the value of the $\Delta x$ factor is close to zero for the optimal $3 \mathrm{U}$ solution. This is consistent with literature. This is mainly due to the fact that a large $\Delta x$ would increase the permeance of all the air barriers (increased $q$-inductance) and also make the rotor flux guides longer and hence a higher reluctance $d$-axis flux path in saturation (premature saturation of the $d$ inductance).

\section{B. Torque and torque ripple of the optimal machines}

The torque waveforms represented in Figs. 7a, 8a and 9a show that the three designs have comparable torque and torque ripple levels at all current loads. The torque ripple is actually non negligible only at $300 \%$ load, but a more significant ripple can be expected with a higher current loading and rate of saturation. Figures $7 \mathrm{~b}, 8 \mathrm{~b}$ and $9 \mathrm{~b}$ report the ripple surfaces over the $d q$ current plane for the three different geometries. All results are comparable, but on a closer look, the six-variables cases 3C and I2U (Figs. 7b and 9b) have overall a lower ripple with respect to the seven variables case $3 \mathrm{U}$ (Fig. 8b). As introduced in subsection III.A, all the final designs have a minimum ripple area around the MTPA condition at $200 \%$ current.

\section{Effect of the number of inputs}

From the results of Figs. 7 to 9 the following conclusions can be drawn:

- the I2U geometry can achieve the same performance of the $3 \mathrm{C}$ case. The rotor topmost steel segment (ie. on top of the $q$ axis and the one that tends to disappear when $\Delta x=0$ ) has little or no impact on performance (see Fig. 10). This is even more pronounced with the circular barriers.

- The I2U solution improves the mechanical strength of the laminations as the mass on the periphery supported by the ribs is reduced and also reduces the moment of inertia. Moreover, there is more room for the shaft.

- The lower performance of the $3 \mathrm{U}$ with respect to its subcase I2U is accountable to the slower convergence of the MOGA when the additional input $\Delta x$ is added. We used the same number of evaluations (3000) and then the same computational time for the two, but the $3 \mathrm{U}$ case would require more evaluations to equal and maybe improve the performance of the $\mathrm{I} 2 \mathrm{U}$ case.

- All considered, the additional degree of freedom $\Delta x$ gives little or no improvement to the performance while slowing the convergence to the optimal solution. The best tradeoff between MOGA time and results for the 3U geometry is then the I2U machine.

\section{Improvement of the Local Search stage}

This section addresses two possible refinements of the LS stage towards the aim of improving the torque ripple at current overload at the expense of a reasonable extra calculation. Reference is made to the I2U geometry, which is the most promising candidate for future developments of SyR and PMassisted SyR designs.

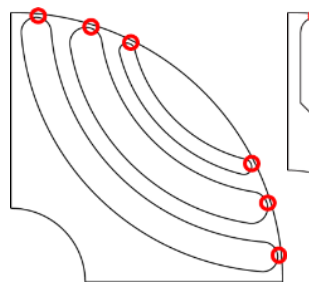

(a) (b)

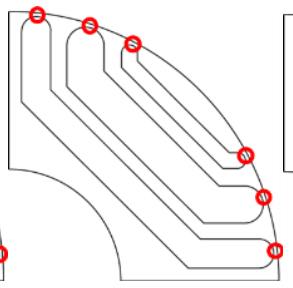

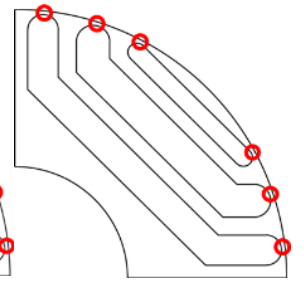

(c)
Fig. 6. MOGA designed rotors. a) Round barriers (3C); b) 3U angled barriers, with the displacement factor $\Delta x$ optimized; c) I2U barriers.

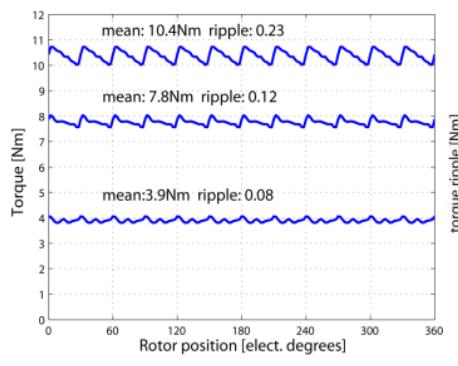

(a)

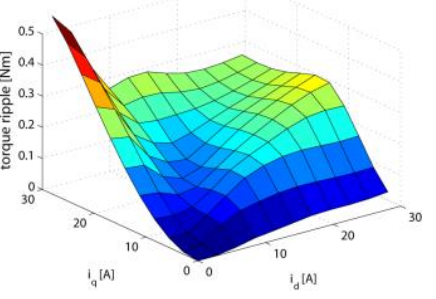

(b)
Fig. 7. 3C optimal machine. a) Torque waveforms at $100 \%$, $200 \%$ and $300 \%$ of rated current. b) Torque ripple surface

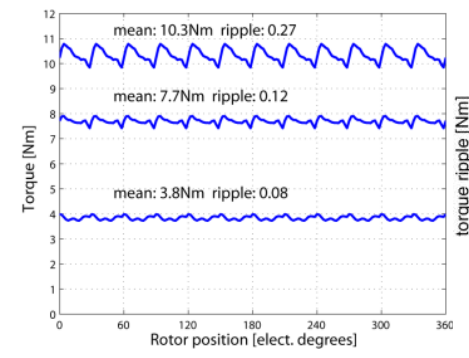

(a)

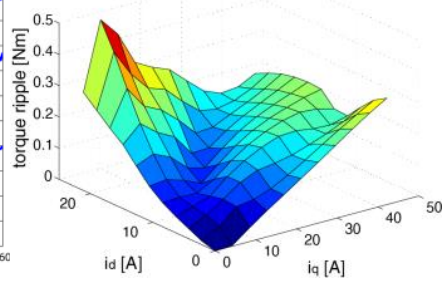

(b)
Fig. 8. 3U optimal machine. a) Torque waveforms at $100 \%$, $200 \%$ and $300 \%$ of rated current. b) Torque ripple surface 


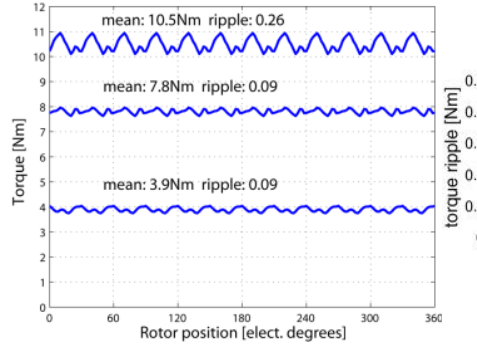

(a)

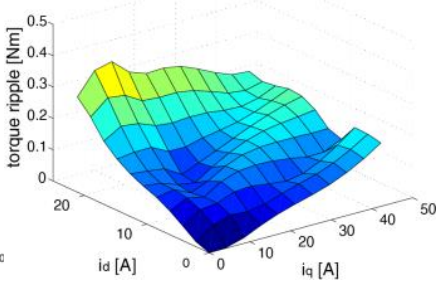

(b)

Fig. 9. I2U optimal machine. a) Torque waveforms at $100 \%$, $200 \%$ and $300 \%$ of rated current. b) Torque ripple surface

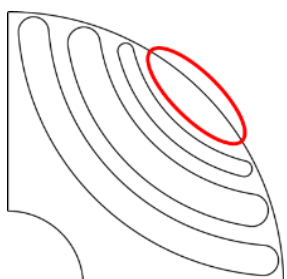

Fig. 10. Main difference between the 3C optimal rotors.

Starting from the same set of GS solutions used for the I2U design of Fig. 2, two directions are explored and then compared with the result of the normal Local Search. The first attempt is called LS15, as the MOGA evaluates the torque ripple over 15 rotor positions instead of 5 in the LS stage. As mentioned, five positions was the default used so far for both GS and LS. As before, the optimization is run at $200 \%$ current load. This LS15 runs requires 7.5 hour, that is 5 hours extra with respect to the standard 2.5 hours LS run. In Fig. 11a it can be seen that the torque ripple is minimized at exactly $200 \%$ current in MTPA conditions (there is a depression in the ripple surface), but not elsewhere: the optimization is too localized.

This is an interesting result as it shows how sensitive the final result is to the conditions simulated during optimization. The ideal optimization should evaluate the machine performance at different load condition, so to avoid the over-emphasis of the final result as in Fig. 11a. However, simulating the machine at more than one load level is unfeasible in terms of computational effort.

The second approach presented here is called LS5+5 and obtains better results even if the required extra-time is lower than that of the LS15 method. The 5+5 approach consists of optimizing the torque and torque ripple at two different current levels. The torque waveform of each candidate is evaluated over 5 positions at two different loads: $100 \%$ and 300\%. Average torque and torque ripple (expressed as a percentage of the average torque) obtained at the two current levels considered are added so to have only two objectives to be optimized via the Pareto front by the optimization algorithm. The LS5+5 takes 5 hours overall and then 2.5 hours of extra time.

In Fig. 11 the torque ripple surfaces over the $i_{d}, i_{q}$ plane are represented for the LS15 and the LS5+5 solutions, respectively. In both cases, the overall ripple performance show an improvement with respect to the I2U machine without LS refinements as represented in Fig. 9. With respect to the comparison between the two LS refinement methods, the surface in Fig 11b (LS5+5) is more regular than the one in Fig 11a (LS15), which shows a very localized ripple minimum, corresponding to $200 \%$ and MTPA, but a worse performance over all the operating range. Besides having a low ripple all over the working plane, the LS5+5 solution has also a minimumripple trajectory that coincides with the MTPA trajectory in the $i_{d}, i_{q}$ plane, which is one of the features of the proposed method.

The torque waveforms of the LS15 and LS5 +5 solutions are represented in Fig. 12, for three different current amplitudes and MTPA conditions. The torque waveforms confirms that the LS15 refinement (Fig. 12a) optimizes the ripple very finely at $200 \%$ load, but not elsewhere. Another major result of this section is that the torque ripple at $300 \%$ overload is very good in both cases, better than the ones of all machines obtained with the standard LS procedure, reported in Figs. 7 to 9. Yet, the average torque values of Fig. 12 are unchanged with respect to the ones in Fig. $7-9$, meaning that the standard LS stage is accurate enough for the average torque goal, while the more challanging goal of minimizing the torque ripple benefits from further optimization refinement. The experimental results in section $\mathrm{V}$ show that when the torque ripple is extremely low in simulation, the results are then not consistent with the experiments, and vice-versa. Having said that, the LS refinements are quite easy to implement and fast computationally and do not harm the final design, even if they improve the rotor design less than expected from the FEA simulations.

\section{EXPERIMENTAL VERIFICATION}

Three prototypes have been fabricated for validating the results of the FEA analysis. The three machines have a common stator, represented in Fig. 13, having 24 slots and distributed windings. The main ratings of the prototypes are reported in Table III. Although it could be possible to extend the design optimization also to the stator, the design of the rotor flux barriers is only considered here, being the most controversial and less standardized point in the design of such kind of machines.

The three rotors are one $3 \mathrm{C}$ and one $\mathrm{I} 2 \mathrm{U}$ solutions, plus a further one representative of a state-of-the-art design technique [5], used as the baseline for the comparison of the automatic design performance. This latter rotor is indicated with the acronym SOA (state-of-the-art). The pictures of the rotor lamination stacks are reported in Fig. 14. The 3C machine is the one obtained with the 4GS+LS automatic procedure, as already reported in Figs. 6a and 10a. The I2U machines is the one obtained with the 4GS plus LS5+5 procedure, described at subsection IV.D and whose laminations are similar to the ones in Figs. 6c and 10b. 


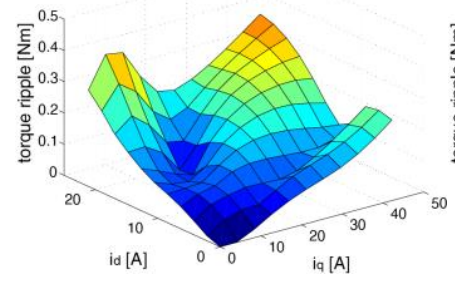

(a)

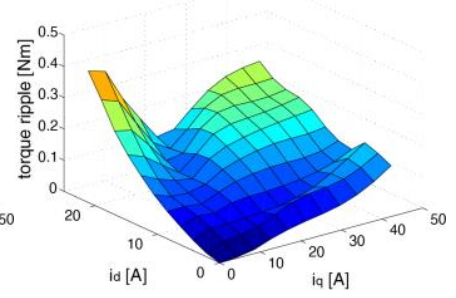

(b)
Fig. 11. Examples of Local Search refinement: a) LS15, torque evaluated over 15 positions; b) LS5+5, torque evaluated over 5 positions and two current amplitudes.

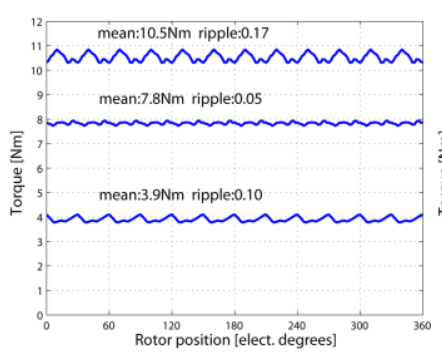

(a)

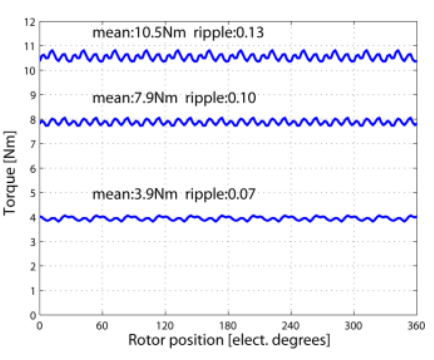

(b)
Fig. 12. Torque waveforms of the two motors at Fig. 7, at 100\%, $200 \%$ and $300 \%$ of rated current.

TABLE III

MAIN PARAMETERS OF PROTOTYPES

\begin{tabular}{ll}
\hline \hline \multicolumn{1}{c}{ Quantity } & Value \\
\hline Stator slots & 24 \\
Pole pairs & 2 \\
Rotor diameter & $58.58 \mathrm{~mm}$ \\
Stator diameter & $101 \mathrm{~mm}$ \\
Stack length & $65 \mathrm{~mm}$ \\
Airgap & $0.5 \mathrm{~mm}$ \\
Rated current (pk) & $13.6 \mathrm{~A}$ \\
Rated voltage (dc-link) & $300 \mathrm{~V}$ \\
Maximum speed & $8000 \mathrm{rpm}$ \\
\hline \hline
\end{tabular}

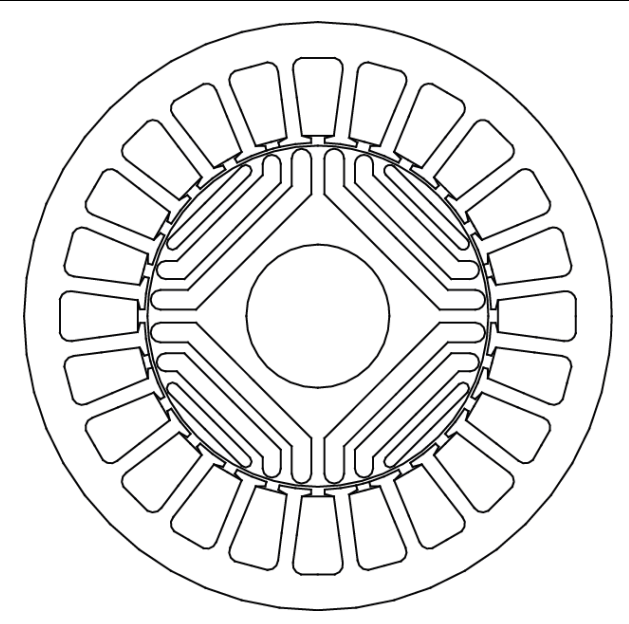

Fig. 13. Cross section of the I2U prototype laminations. The stator is common to all the prototypes

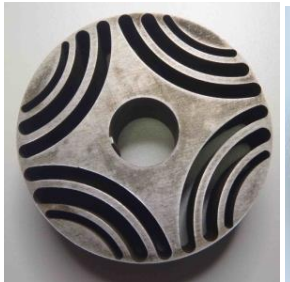

(a)

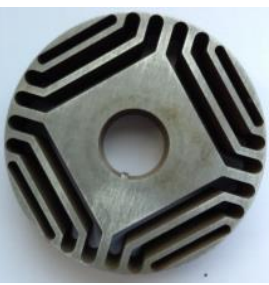

(b)

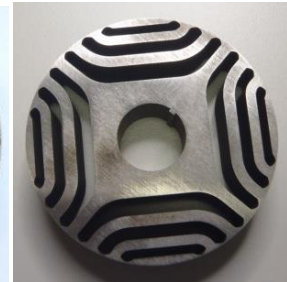

(c)
Fig. 14. Rotor laminations of prototype 3C (a), prototype I2U (b) and the state-of-the-art solution SOA (c).

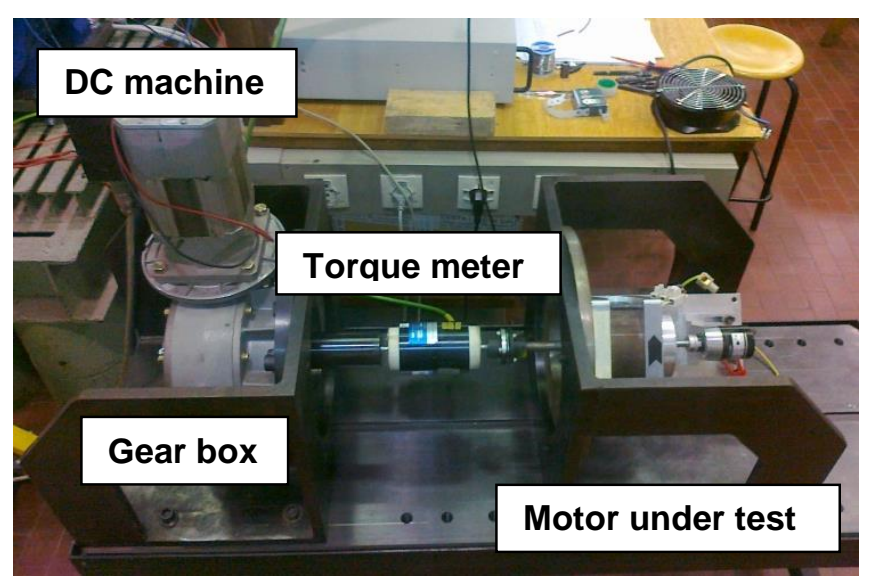

Fig. 15. Test bench used to measure the torque ripple maps of the motor prototypes.

\section{A. Experimental setup}

A dedicated test bench is used to measure the torque waveform of the prototypes for different $i_{d}, i_{q}$ current combinations. A speed-controlled DC motor having very low torque ripple drives the motor under test via a reduction gearbox. The constant test speed is $10 \mathrm{rpm}$. The torque is measured using a high precision torque meter. The motor under test is vector controlled, using a dSPACE 1104 board. The $i_{d}, i_{q}$ reference sequence and the acquisition of the torque signal during one motor revolution are automatically handled by means of a Matlab script using the commands of the MLIB/MTRACE dSPACE library [22] for dSPACE experiment automation. The torque meter maximum rating is $10 \mathrm{Nm}$ which corresponds to an area of operation enclosed by $i_{d}=20 \mathrm{~A}, i_{q}=30 \mathrm{~A}$ in the d,q plane. The test bench is shown in Fig. 15.

\section{B. Experimental results}

At first, the average torque performance is considered. The measured torque versus current phase angle curves of the three motors are represented in Fig. 16. Three current amplitudes are represented, corresponding to $48 \%, 123 \%$ and $239 \%$ of the continuous current level. Phase angle zero means that the current vector is aligned with the $d$-axis, whereas phase angle $90^{\circ}$ corresponds to the $q$-axis. The three prototypes are pretty comparable, and the SOA has a little advantage at current overload, commented hereafter.

The measured torque values are compared with the FEA calculated ones on the graphs in Figs. 17 to 20. For all the machines the discrepancy between calculations and experiments is very little with the FEA results showing slightly higher torque for all prototypes. The SOA motor curves (Fig. 19) show practically no error between FEA and experiments, 
while for the I2U motor the discrepancy is a bit higher than for the $3 \mathrm{C}$ one: the two automatic designs were forecast to give the same torque at all conditions by FEA, and this little discrepancy is likely to be justified through manufacturing and material properties' tolerances. More comments about potential FEA discrepancies are given in the following paragraphs. A first conclusion is that the automatic designs are competitive with the SOA benchmark in terms of torque, although their rotor geometries are relatively very simple and with a reduced number of geometric design degrees of freedom. The little advantage of the SOA motor at saturated conditions, also confirmed by FEA, is justified by the fact that such rotor was designed through approximately twenty degrees of freedom instead of the six or seven for the automatic designs presented. The SOA machine has a more refined compromise between the thickness of the air insulation layers and the steel flux guides.

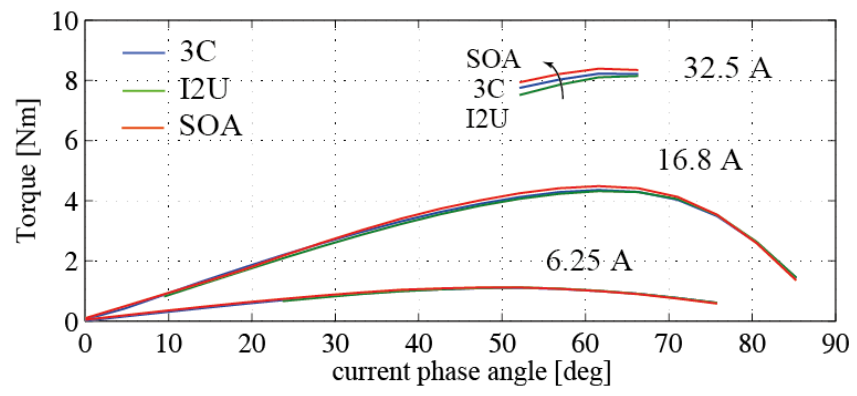

Fig. 16. Comparison of the measured average torque as a function of the current phase angle at different current amplitudes for the three prototypes.

The steel guides of Fig. 14c are evidently thicker than the ones of the MOGA-based designs in Figs. 14a and 14b, but the isolation is still good due to the greater degrees of freedom in the geometrical shape of the barriers. Such an amount of design freedom is however impractical for an automatic design environment as the cost to benefit ratio in terms of computational time and performance when compared to the proposed design methodology is clearly poor.

Examples of torque waveforms are reported in Figs. 20 to 22 for the three prototypes. Results over one electrical period (half mechanical revolution) are shown and the same three current levels of the torque versus current phase graphs are used here. The FEA and measured values are directly compared. The experiments confirm that the torque ripple of the automatic designs is fairly minimized and it is lower than the one of the SOA design, at least within the rated current range. Unexpectedly, at high overload the two automatic designs (3C in Fig. 20, I2U in Fig. 21) have a $12^{\text {th }}$ harmonic component which is stronger than that expected from the FEA results.

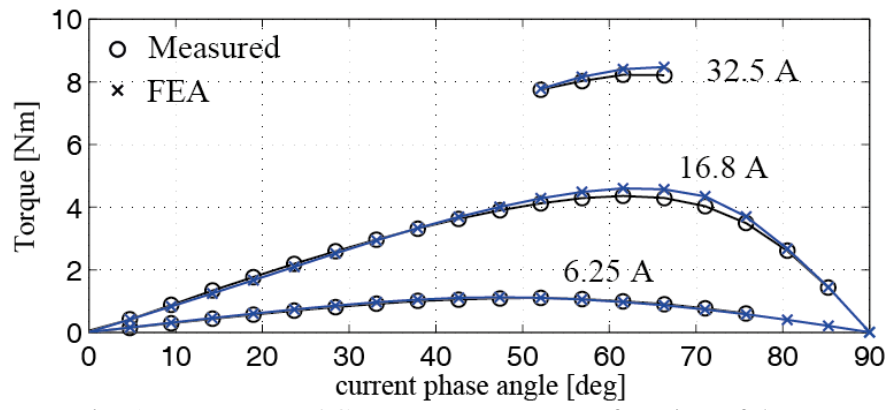

Fig. 17. Prototype 3C: average torque as a function of the current phase angle, at different current amplitudes.

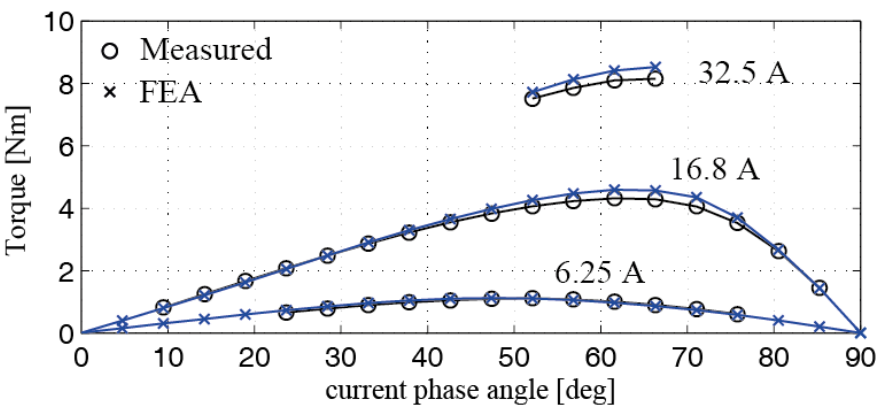

Fig. 18. Prototype I2U: average torque as a function of the current phase angle, at different current amplitudes.

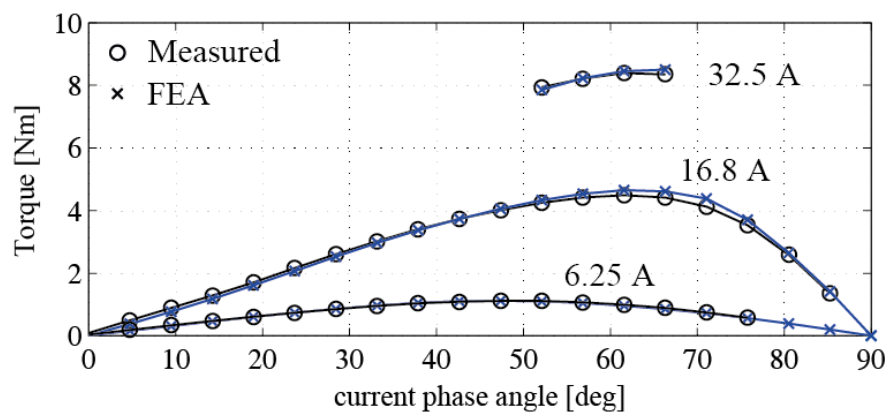

Fig. 19. Prototype SOA: average torque as a function of the current phase angle, at different current amplitudes.

This harmonic order corresponds to the stator slots periodicity which is actually the one minimized by the MOGA. The experimental results indicate that the ripple minimization is lower than the one predicted by the FEA. In fact, the lower the expected $12^{\text {th }}$ harmonic component, the higher it is the experimental discrepancy. Ironically, the I2U motor (Fig. 21), which is more finely-optimized via the LS5+5 run sees its torque at overload to be the least compliant with the FEA waveform, whereas the SOA design has nearly no discrepancy. The authors' understanding is that the discrepancy is the result of manufacturing tolerances and material properties' uncertainties. The automatic designs have thin, optimized flux guides, whereas the flux guides of the SOA design are thicker. This fact makes the two automatic designs more sensitive to lamination fabrication tolerances (e.g. the flux guide maybe slightly thinner or thicker than expected) and to the uncertain knowledge of the saturated B-H curve of the laminations (the grade of saturation in the thinner barriers is higher). Additional prototype construction and the custom identification of the B-H curves for the lamination samples used, including the effect of lamination cutting, are currently being considered to clarify this 
point on a quantitative basis.

Having said this, the experimental results clearly demonstrate the strong expected improvement in the torque ripple of the automatic designs (Figs. 20 and 21) when compared to that of the SOA motor (Fig. 22), at all loads. Figures 23 to 25 report the performance of the torque ripple over the $i_{d}-i_{q}$ plane for FEA (subfigures a) and measurement (subfigures b) for the three prototypes. As demonstrated already for the average torque, the torque ripple surfaces also show a general good agreement between FEA and experiment. For all the machines, the ripple tends to grow with the $i_{q}$ current component, i.e. with load torque. As already commented, the torque ripple of the automatic designs (Figs. 23-24) is underestimated by the FEA at higher loads, i.e. at higher values of $i_{q}$, while the SOA prototype has a very fair correspondence between FEA and measurement. The automatic designs (3C in Fig. 23, I2U in Fig. 24) have a V-shaped ripple surface, with a depression in the area of the $i_{d}-i_{q}$ plane where the MTPA trajectory is. This validates the particularly advantageous performance of MOGA designed machines as explained in subsection III.A and in Fig. 11. This is not the case with the SOA design (Fig. 25), where the ripple grows monotonically with $i_{q}$, independently from $i_{d}$. There is no example in literature of MTPA-specialized ripple minimization and this is too farfetched to do using any analytical method to reproduce such results. This achievement was not in the original goals of this analysis, but it is yet one of the original contributions of the MOGA and FEA based design presented in the paper.

\section{CONCLUSION}

The paper presents a procedure for the automatic design of multi-layer synchronous reluctance rotors based on MultiObjective GA optimization and FEA. The guidelines for the fast use of FEA and MOGA were described in terms of MOGA settings and FEA evaluation of SyR motors. Different barrier geometries were analyzed and compared. A state of the art rotor, designed for the same stator, was manufactured and used as a baseline for comparison. The analysis, confirmed by experiments, shows that the SyR motors can be designed automatically within a reasonable time, having a comparable torque density and a lower torque ripple. Moreover, it was shown that the number of degrees of freedom of the rotor geometry that plays a key role is limited and not all degrees of freedom are helpful. This conclusion led to exclude the generic $3 \mathrm{U}$ geometry from the prototyping section in favor of the simpler I2U geometry.

According to the results presented in the paper, a reasonable number of rotor degrees of freedom are two per barrier. This is the same for both the automatically designed prototypes $3 \mathrm{C}$ and I2U. More degrees of freedom can improve the performance at the cost of a longer computation. For the two selected templates, 3C and I2U, they both have the same potential, according to FEA.

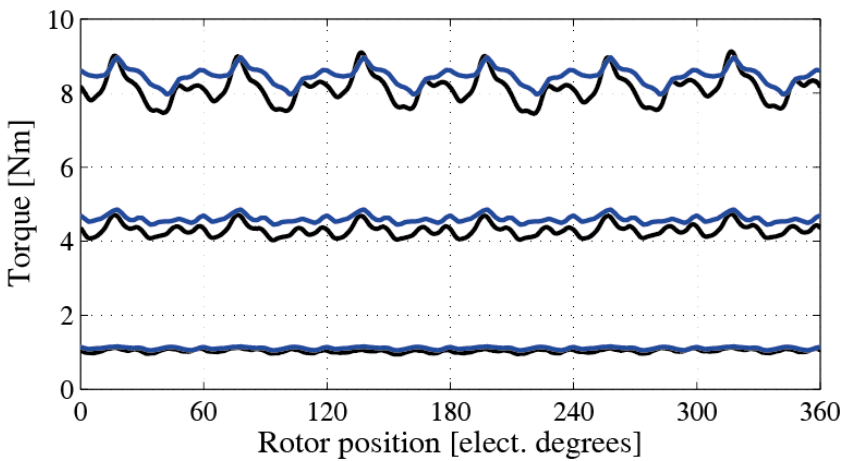

Fig. 20. Prototype $3 \mathrm{C}$ (black lines) and FEA results (blue lines): torque waveforms at $6.25 \mathrm{~A}(48 \%), 16.8 \mathrm{~A}(123 \%)$ and 32.5 A $(239 \%)$ on the MTPA.

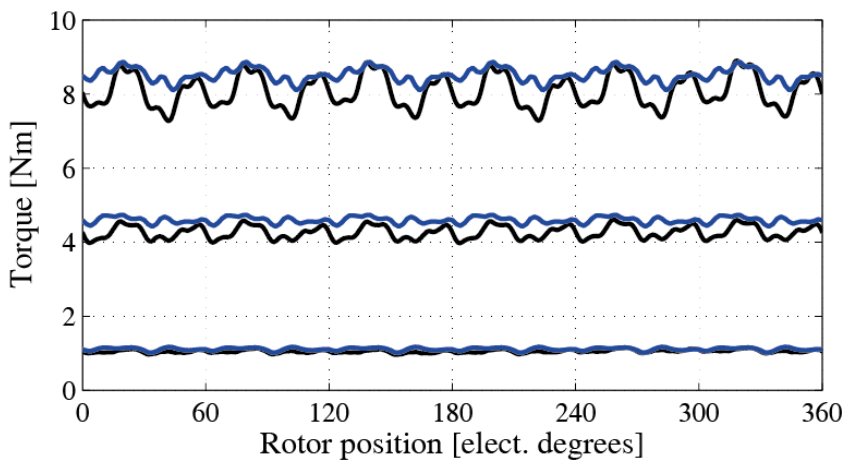

Fig. 21. Prototype I2U (black lines) and FEA results (blue lines): torque waveforms at $6.5 \mathrm{~A}(48 \%), 16.8 \mathrm{~A}(123 \%)$ and 32.5 A $(239 \%)$ on the MTPA.

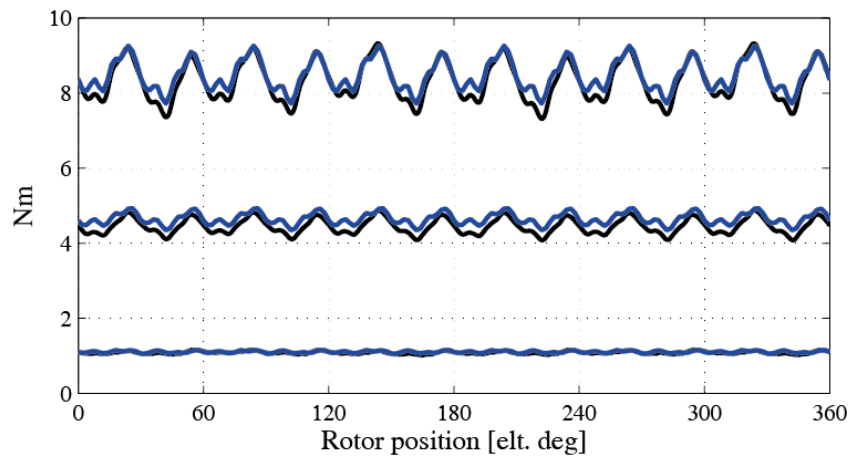

Fig. 22. Prototype SOA (black lines) and FEA results (blue lines): torque waveforms at $6.5 \mathrm{~A}(48 \%), 16.8 \mathrm{~A}(123 \%)$ and 32.5 A $(239 \%)$ on the MTPA.

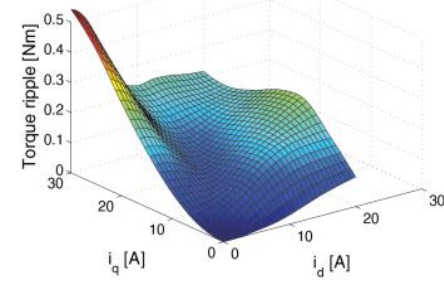

(a)

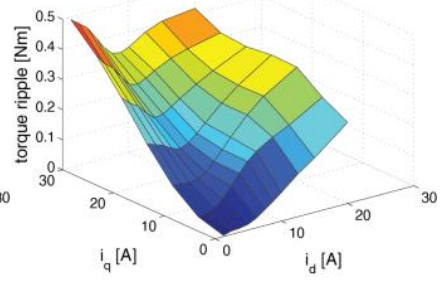

(b)
Fig. 23. Prototype 3C: torque ripple surface over the $i_{d}, i_{q}$ plane, according to FEA (a) and measurements (b). 
(a)

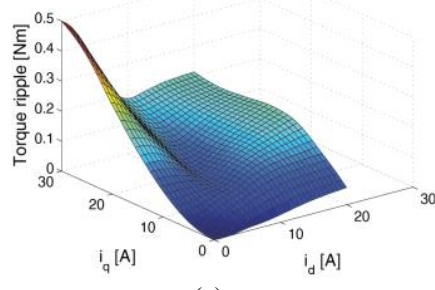

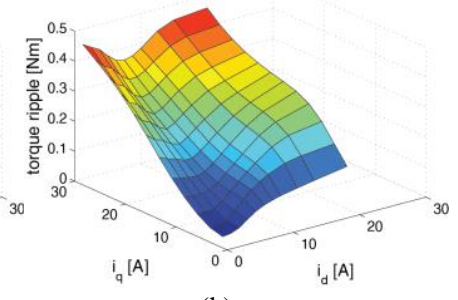

(b)
Fig. 24. Prototype I2U: torque ripple surface over the $i_{d}, i_{q}$ plane, according to FEA (a) and measurements (b).

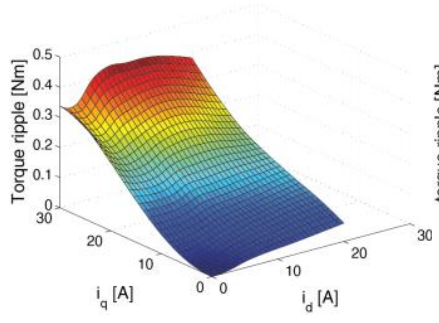

(a)

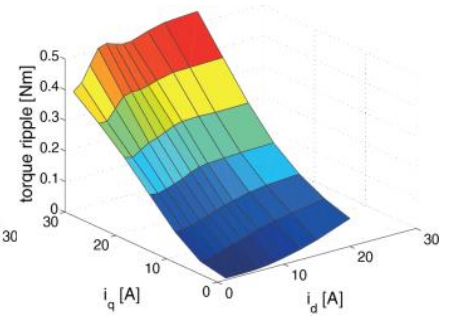

(b)
Fig. 25. Prototype SOA: torque ripple surface over the $i_{d}, i_{q}$ plane, according to FEA (a) and measurements (b).

The I2U shape has a lower inertia, less structural challanges at high speed and it is more suitable for permanent magnet insertion in case of a PM-assisted machine design. Future work will investigate the effects of manufacturing tolerances and the designs' sensitivity to them. The results of the paper show that the SyR motors can be designed automatically and that very simple geometries can match, if not improve, the performance of more complicated rotor geometries in the literature.

\section{ACKNOWLEDGMENTS}

This work was supported in part by project PON MALET code PON01_01693.

\section{REFERENCES}

[1] T. A. Lipo, T. J. E. Miller, A. Vagati, I. Boldea, L. Malesani, and T. Fukao, "Synchronous reluctance drives," in Conf. Rec. IEEE IAS Annu. Meeting, Denver, CO, Oct. 1994.

[2] Staton, D.A.; Miller, T.J.E.; Wood, S.E., "Maximising the saliency ratio of the synchronous reluctance motor," Electric Power Applications, IEE Proceedings B , vol.140, no.4, pp.249,259, Jul 1993

[3] Kamper, M.J.; Van der Merwe, F.S.; Williamson, S.; , "Direct finite element design optimisation of the cageless reluctance synchronous machine," Energy Conversion, IEEE Transactions on , vol.11, no.3, pp.547-555, Sep 1996

[4] Boldea, Ion. Reluctance synchronous machines and drives. Oxford,, UK: Clarendon Press, 1996.

[5] Vagati, Alfredo. "Synchronous reluctance electrical motor having a low torque-ripple design." U.S. Patent No. 5,818,140. 6 Oct. 1998.

[6] RR Moghaddam, "Rotor for a Synchronous Reluctance Machine", US Patent App. 13/230,543, 2011

[7] W. Soong and T. J. E. Miller, "Field weakening performance of brushless synchronous AC motor drives," Proc. IEE-Elect. Power Appl., vol. 141, no. 6, pp. 331-340, Nov. 1994.

[8] Ooi, S.; Morimoto, S.; Sanada, M.; Inoue, Y.; , "Performance evaluation of a high power density PMASynRM with ferrite magnets," Energy Conversion Congress and Exposition (ECCE), 2011 IEEE , vol., no., pp.4195-4200, 17-22 Sept. 2011

[9] Vagati, A.; Canova, A.; Chiampi, M.; Pastorelli, M.; Repetto, M., "Design refinement of synchronous reluctance motors through finite-element analysis," Industry Applications, IEEE Transactions on , vol.36, no.4, pp.1094,1102, Jul/Aug 2000
[10] Lovelace, E.C.; Jahns, T.M.; Lang, J.H.; , "A saturating lumpedparameter model for an interior PM synchronous machine," Industry Applications, IEEE Transactions on , vol.38, no.3, pp.645-650, May/Jun 2002

[11] Sizov, G.Y.; Ionel, D.M.; Demerdash, N.A.O.; , "Multi-objective optimization of PM AC machines using computationally efficient - FEA and differential evolution," Electric Machines \& Drives Conference (IEMDC), 2011 IEEE International, vol., no., pp.1528-1533, 15-18 May 2011

[12] Miller, T.J.E.; Popescu, M.; Cossar, C.; McGilp, M.I.; Olaru, M.; Davies, A.; Sturgess, J.; Sitzia, A., "Embedded Finite-Element Solver for Computation of Brushless Permanent-Magnet Motors," Industry Applications, IEEE Transactions on , vol.44, no.4, pp.1124,1133, Julyaug. 2008

[13] Bianchi, N.; Bolognani, S., "Design optimisation of electric motors by genetic algorithms," Electric Power Applications, IEE Proceedings - , vol.145, no.5, pp.475-483, Sep 1998.

[14] Wen Ouyang; Zarko, D.; Lipo, T.A., "Permanent Magnet Machine Design Practice and Optimization," Industry Applications Conference, 2006. 41st IAS Annual Meeting. Conference Record of the 2006 IEEE, vol.4, no., pp.1905-1911, 8-12 Oct. 2006

[15] Pellegrino, G.; Cupertino, F., "FEA-based multi-objective optimization of IPM motor design including rotor losses," Energy Conversion Congress and Exposition (ECCE), 2010 IEEE, vol., no., pp.3659-3666, 12-16 Sept. 2010

[16] Cupertino, F.; Pellegrino, G.; Armando, E.; Gerada, C., "A SyR and IPM machine design methodology assisted by optimization algorithms" Energy Conversion Congress and Exposition (ECCE), 2012 IEEE , vol., no., 15-20 Sept. 2012.

[17] J. Legranger, G. Friedrich, S. Vivier, and J.C. Mipo, "Combination of Finite-Element and Analytical Models in the Optimal Multidomain Design of Machines: Application to an Interior Permanent-Magnet Starter Generator", IEEE Transactions on Industry Applications, Vol. 46, n. 1, pp. 232-239, Jan/Feb 2010.

[18] Y. Duan, and D.M. Ionel, "A Review of Recent Developments in Electrical Machine Design Optimization Methods With a PermanentMagnet Synchronous Motor Benchmark Study", IEEE Transactions on Industry Applications, Vol. 49, n. 3, pp. 1268-1275, May/June 2013.

[19] David Meeker, "Finite Element Method Magnetics", Ver. 4.2 User's Manual, February 5, 2009, [Online] available: http://www.femm.info/Archives/doc/manual.pdf

[20] http://www.mathworks.com

[21] K. Deb, A. Patrap, S. Agarwal, and T. Meyarivan: "A Fast and Elitist Multiobjective Genetic Algorithm: NSGA-II", IEEE Transactions on Evolutionary Computation, vol. 6, n. 2, April 2002, pp. 182-197.

[22] http://www.dspace.com

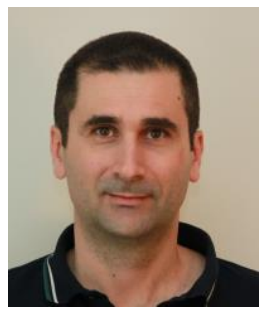

Francesco Cupertino (M'08, SM'12), received the Laurea degree and the $\mathrm{PhD}$ degree in Electrical Engineering from the Politecnico di Bari, Italy, in 1997 and 2001 respectively. From 1999 to 2000 he was with PEMC research group, University of Nottingham, UK. Since July 2002 he is an Assistant Professor at the Politecnico di Bari. His research interests include the design of permanent magnet electrical machines, intelligent motion control of electrical machines, and applications of computational intelligence to control and design. He is the author or co-author of more than 100 scientific papers on these topics. He is the scientific director of the laboratory Energy Factory Bari (EFB), a joint initiative of the Politecnico di Bari and GE AVIO, aimed at developing research projects in the fields of aerospace and energy.

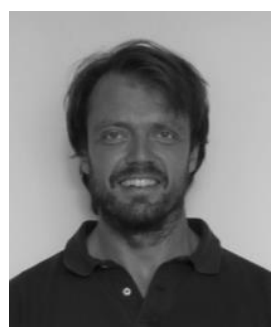

Gianmario Pellegrino (M'06, SM'13), received the M.Sc. and Ph.D. degrees in electrical engineering from Politecnico di Torino, Turin, Italy, in 1998 and 2002, respectively. Since 2002 he is with Politecnico di Torino. His research interests include the design of electrical machines and the control of electrical drives. $\mathrm{He}$ is involved in research projects with industry and has more than 20 journal papers and one patent. Dr. Pellegrino is an Associate Editor for the IEEE Transactions on Industry Applications and an IEEE Senior Member. He is the co-recipient of three Prize Paper Awards. He 
was a guest researcher at Aalborg University, Denmark, in 2002, a visiting fellow at Nottingham University, UK, in 2010/2011, and an honorary fellow at the University of Wisconsin-Madison, USA, in 2013.

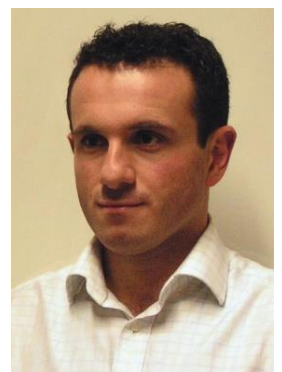

Prof. Chris Gerada (M'05) obtained his $\mathrm{PhD}$ in Numerical Modelling of Electrical Machines from the University of Nottingham, UK in 2005. He subsequently worked as a researcher at the University of Nottingham on high performance electrical drives and on the design and modelling of electromagnetic actuators for aerospace applications. He was appointed as Lecturer in Electrical Machines in 2008, an Associate Professor in 2011 and Professor in 2013. His core research interests include the design and modelling of high performance electric drives and machines. Prof. Gerada has been the project manager of the GE Aviation Strategic Partnership since 2006 and in 2011 was awarded a Royal Academy of Engineering Senior Research Fellowship supported by Cummins. He is also an Associate Editor for the Transactions in Industry Applications and executive member of the management board of the UK Magnetic Society and the IET Aerospace Technical and Professional Network. 